\title{
Microwave-Assisted Synthesis and Antimicrobial Activity of Some Novel Isatin Schiff Bases Linked to Nicotinic Acid via Certain Amino Acid Bridge
}

\author{
Ahmed M. Naglah, ${ }^{1,2}$ Hassan M. Awad, ${ }^{3}$ Mashooq A. Bhat, ${ }^{4}$ \\ Mohamed A. Al-Omar, ${ }^{1}$ and Abd El-Galil E. Amr ${ }^{1,5}$ \\ ${ }^{1}$ Department of Pharmaceutical Chemistry, Drug Exploration \& Development Chair, College of Pharmacy, \\ King Saud University, Riyadh 11451, Saudi Arabia \\ ${ }^{2}$ Peptide Chemistry Department, Chemical Industries Research Division, National Research Centre, Dokki, Cairo 12622, Egypt \\ ${ }^{3}$ Chemistry of Natural and Microbial Products Department, Pharmaceutical Industries Division, National Research Centre, \\ Dokki, Cairo 12622, Egypt \\ ${ }^{4}$ Department of Pharmaceutical Chemistry, College of Pharmacy, King Saud University, Riyadh 11451, Saudi Arabia \\ ${ }^{5}$ Applied Organic Chemistry Department, National Research Centre, Dokki, Cairo 12622, Egypt \\ Correspondence should be addressed to Ahmed M. Naglah; amnaglah@gmail.com
}

Received 1 October 2014; Revised 30 November 2014; Accepted 21 December 2014

Academic Editor: Rabah Boukherroub

Copyright (C) 2015 Ahmed M. Naglah et al. This is an open access article distributed under the Creative Commons Attribution License, which permits unrestricted use, distribution, and reproduction in any medium, provided the original work is properly cited.

The coupling reaction of nicotinic acid with certain L-amino acid methyl esters including valine, leucine, and phenylalanine was done by the use of acid chloride method. The products were reacted with hydrazine hydrate $99 \%$ to give the corresponding hydrazides that were reacted with indoline-2,3-dione (isatin) to get Schiff bases under the application of microwave irradiation technique. These novel compounds were characterized by means of their FT-IR, ${ }^{1} \mathrm{H}$ NMR, and mass spectral data. Additionally, the specific optical rotation and elemental analysis were measured. The in vitro antimicrobial activity of the synthesized compounds was evaluated by agar diffusion method. The compounds showed a strong antimicrobial inhibitory activity. Most of the test compounds possessed a broad spectrum of activities having MIC values ranging from $50 \mu \mathrm{g} / \mathrm{mL}$ to $500 \mu \mathrm{g} / \mathrm{mL}$.

\section{Introduction}

Nicotinic acid (pyridine-3-carboxylic acid), also known as niacin and vitamin $\mathrm{B} 3$, is found in various plants and animals; also, it has vital roles in such biological processes as production of energy [1]. Nicotinic acid derivatives and its isomers have antibacterial, antioxidant, anti-inflammatory, anticarcinogenic, and antitubercular activities [2], signal transduction, regulation of gene expression [3], and involvement in the synthetic pathway of lipids [4]. On the other hand, some of new heterocyclic and peptide derivatives have been studied with respect to antivirus [5], anti-inflammatory [6], enzymatic peptide [7], and antimicrobial activities [8,9]. Also, there are several successful publications of microwaveassisted solid phase peptide synthesis of various unnatural biopolymers such as peptoids, pseudopeptides, small peptides [10], phosphopeptides [11], difficult peptides [12], $\beta$ peptide libraries [13], and glycopeptides [14].

It is as well known that isatin is an endogenous compound identified in humans and that its biological properties include a range of actions in the brain; it offers protection against certain types of infections [15]. Isatin constitutes an important class of bioactive compounds exhibiting caspase inhibitor $[16,17]$, antiproliferative [18], and antibacterial activities [19]. Furthermore, novel inhibitors of mycobacterium tuberculosis, prepared from indoline-2,3-dione (isatin) derivatives by the use of microwave irradiation technique, have been reported [20].

Due to the importance of nicotinic acid derivatives, amino acids, peptides, and isatin as bioactive compounds and 
in continuation of our previous works in heterocyclic and peptide chemistry [21-26], the aim of this study is to prepare novel antimicrobial compounds that have crucial importance in the way of overcoming the remarkable adaptability of the bacteria.

\section{Experimental}

\subsection{Chemistry}

2.1.1. General. The organic solvents and the chemicals used in this part were obtained from Sigma (USA) and Fluka (Switzerland) chemical companies and the used amino acids are of L-configuration. Microwave irradiations were carried out using a domestic microwave oven LG-MS-2044 W/OO, with frequency of $2450 \mathrm{MHz}$ and operating at 420 watts of the total power. Infrared (IR) spectra were recorded as $\mathrm{KBr}$ disks using the Perkin Elmer FT-IR Spectrum BX Apparatus located at the Research Center, College of Pharmacy, King Saud University (Riyadh, Saudi Arabia). Melting points were determined in opened glass capillary tubes with an "Electrothermal" Digital Melting Point Apparatus (model: IA9100) and are uncorrected. Elemental microanalysis for carbon, hydrogen, and nitrogen was measured at the Microanalytical Unit, National Research Centre (Cairo, Egypt), and was found within the acceptable limits of the calculated values $( \pm 0.4 \%)$. Specific optical rotations were measured at the National Research Centre (Cairo, Egypt) with "A. KRUSS, OPTRONIC, P8000" polarimeter (Germany), in a $1 \mathrm{dm}$ length for the observation tube, in methanol as a solvent. NMR spectra were scanned in DMSO- $d_{6}$ on a Bruker NMR spectrophotometer operating at $500 \mathrm{MHz}$ for ${ }^{1} \mathrm{H}$ at the Research Center, College of Pharmacy, King Saud University (Riyadh, Saudi Arabia). Chemical shifts are expressed in $\delta$-values (ppm) relative to tetramethylsilane (TMS) as an internal standard. Coupling constants $(J)$ are expressed in $\mathrm{Hz} . \mathrm{D}_{2} \mathrm{O}$ was added to confirm the exchangeable protons. High resolution mass spectra were measured on a JEOL M Station JMS-700 system at the Research Center, College of Pharmacy, King Saud University (Riyadh, Saudi Arabia). Analytical thin layer chromatography (TLC) was performed on silica gel aluminum sheets, $60 \mathrm{~F}_{254}$ (E. Merck).

2.1.2. Synthesis of Esters $(\mathbf{3} \boldsymbol{a}-\boldsymbol{c})$. These compounds were prepared by the reaction of acid chloride 1 with amino acid esters 2a-c according to the reported method [27].

2.1.3. Synthesis of Hydrazide Derivatives $(\mathbf{4 a}-\boldsymbol{c})$. Hydrazine hydrate $99 \%(0.8 \mathrm{~mL}, 16 \mathrm{mmol})$ was added to a methanolic solution $(10 \mathrm{~mL})$ of $3 \mathbf{a}-\mathbf{c}(1 \mathrm{mmol})$. The reaction mixture was refluxed for $10 \mathrm{~h}$, after which the solvent was evaporated under reduced pressure. The obtained residue was triturated with ether, filtered off, and crystallized to afford the corresponding hydrazides $(\mathbf{4 a - c})$. The physical properties and spectral data of compounds $N$-(1hydrazinyl-3-methyl-1-oxobutan-2-yl)nicotinamide (4a) and
$N$-(1-Hydrazinyl-1-oxo-3-phenylpropan-2-yl)nicotinamide (4c) were identical with those reported in [28].

$N$-(1-Hydrazinyl-4-methyl-1-oxopentan-2-yl)nicotinamide (4b). Yield (66\%); m. p. $182-185^{\circ} \mathrm{C} ;-[\alpha]_{\mathrm{D}}^{25}=-80(c=0.02$, $\mathrm{MeOH})$; IR (KBr) $v_{\max } / \mathrm{cm}^{-1} 3413-3276(\mathrm{NH}), 1685(\mathrm{C}=\mathrm{O})$, $1593(\mathrm{C}=\mathrm{N}) ;{ }^{1} \mathrm{H}$ NMR (DMSO- $\left.d_{6}\right) \delta$ 0.87-0.92 (m, 6H, $\left.2 \mathrm{CH}_{3}\right), 1.52-1.53\left(\mathrm{~m}, 1 \mathrm{H},-\mathrm{CH}\left(\mathrm{CH}_{3}\right)_{2}\right), 1.64-1.69(\mathrm{~m}, 2 \mathrm{H}$, $-\mathrm{CH}_{2}$ ), 4.31 (br. s, $2 \mathrm{H}, \mathrm{D}_{2} \mathrm{O}$ exchangeable, $\mathrm{NH}_{2}$ hydrazide), 4.50-4.51 (m, 1H, -NH-C-ㅡ-CO), 7.5 (s, 1H, pyridine $\mathrm{H} 5$ ), $8.23(\mathrm{~s}, 1 \mathrm{H}$, pyridine $\mathrm{H} 4), 8.71\left(\mathrm{~s}, 2 \mathrm{H}, \mathrm{D}_{2} \mathrm{O}\right.$ exchangeable, $\mathrm{NH}$ amide $+\mathrm{NH}$ hydrazide), 9.04 ( $\mathrm{s}, 1 \mathrm{H}$, pyridine $\mathrm{H} 6)$, 9.29 (s, 1H, pyridine H2); HR-MS: 250.3587. Anal. Calcd. for $\mathrm{C}_{12} \mathrm{H}_{18} \mathrm{~N}_{4} \mathrm{O}_{2}$ (250.2969): $\mathrm{C}$ (57.58), $\mathrm{H}$ (7.25), $\mathrm{N}$ (22.38). Found: C (57.54), H (7.24), N (22.35).

2.1.4. Synthesis of Isatin Hydrazones $(\mathbf{6} \boldsymbol{a}-\mathbf{g})$ Using Microwave Radiation. A solution of isatin $5 \mathbf{a}-\mathbf{c}(1 \mathrm{mmol})$ and hydrazide 4a-c $(1 \mathrm{mmol})$ in ethanol $(15 \mathrm{~mL})$ was prepared. Few drops of glacial acetic acid were added and whole reaction mixture was irradiated under microwave irradiation at 420 watts ( $110^{\circ} \mathrm{C}$ ) for $5-10$ minutes. The reaction mixture was cooled. Then, the solid separated on cooling was filtered, washed with cold ethanol, dried, and recrystallized from the appropriate solvent to obtain hydrazones $\mathbf{6 a - g}$.

N-(1-(2-(5-Chloro-2-oxoindolin-3-ylidene)hydrazinyl)-3-methyl-1-oxobutan-2-l)nicotinamide (6a). Yield (69\%); m. p. $210-214^{\circ} \mathrm{C} ;-[\alpha]_{\mathrm{D}}^{25}=-48(c=0.01, \mathrm{MeOH}) ; \operatorname{IR}(\mathrm{KBr})$ $\nu_{\max } / \mathrm{cm}^{-1} 3410(\mathrm{NH}), 1690(\mathrm{C}=\mathrm{O}), 1618(\mathrm{C}=\mathrm{N}) ;{ }^{1} \mathrm{H}$ NMR $\left(\mathrm{DMSO}-d_{6}\right) \delta 0.98-1.04\left(\mathrm{~m}, 6 \mathrm{H}, 2 \mathrm{CH}_{3}\right), 1.90-1.91(\mathrm{~m}, 1 \mathrm{H}$, $\left.-\mathrm{CH}\left(\mathrm{CH}_{3}\right)_{2}\right), 4.41-4.47$ (m, 1H, -NH-CH-CO), 5.40-5.41 (m, $1 \mathrm{H},-\mathrm{NH}-\mathrm{CH}-\mathrm{CO}), 6.86-8.08$ (m, 5H, Ar-H), 8.64 (s, $1 \mathrm{H}$, Ar- $\mathrm{H}), 8.93$ (s, 1H, $\mathrm{D}_{2} \mathrm{O}$ exchangeable, $\mathrm{NH}$ amide), 9.17-9.40 ( $\mathrm{m}, 2 \mathrm{H}, \mathrm{Ar}-\mathrm{H}), 11.60$ (s, $1 \mathrm{H}, \mathrm{D}_{2} \mathrm{O}$ exchangeable, $\mathrm{NH}$ isatin), 12.57 ( $\mathrm{s}, 1 \mathrm{H}, \mathrm{D}_{2} \mathrm{O}$ exchangeable, $\mathrm{NH}$ hydrazide), $13.39(\mathrm{~s}, 1 \mathrm{H}$, $\mathrm{D}_{2} \mathrm{O}$ exchangeable, $\mathrm{NH}$ hydrazide); HR-MS: 399.1098. Anal. Calcd. for $\mathrm{C}_{19} \mathrm{H}_{18} \mathrm{ClN}_{5} \mathrm{O}_{3}$ (399.11): $\mathrm{C}$ (57.07), $\mathrm{H}$ (4.54), $\mathrm{N}$ (17.52). Found: C (57.04), H (4.58), N (17.50).

N-(4-Methyl-1-oxo-1-(2-(2-oxoindolin-3-ylidene)hydrazinyl) pentan-2-yl)nicotinamide (6b). Yield (93\%); m. p. 220-222 ${ }^{\circ} \mathrm{C}$; $-[\alpha]_{\mathrm{D}}^{25}=-132(c=0.03, \mathrm{MeOH}) ; \mathrm{IR}(\mathrm{KBr}) \nu_{\max } / \mathrm{cm}^{-1} 3414$ $(\mathrm{NH}), 1700(\mathrm{C}=\mathrm{O}), 1641(\mathrm{C}=\mathrm{N}) ;{ }^{1} \mathrm{H}$ NMR $\left(\mathrm{DMSO}-d_{6}\right) \delta$ 0.92-1.10 (m, 6H, 2CH $), 1.62-1.92\left(\mathrm{~m}, 3 \mathrm{H},-\mathrm{CH}\left(\mathrm{CH}_{3}\right)_{2}+\right.$ $\left.-\mathrm{CH}_{2}\right), 4.64-4.66(\mathrm{~m}, 1 \mathrm{H},-\mathrm{NH}-\mathrm{CH}-\mathrm{CO}), 5.53-4.54(\mathrm{~m}, 1 \mathrm{H}$, -NH-CH-CO), 6.93-7.56 (m, 5H, Ar-H), 8.28 (s, 1H, Ar-H), $8.76\left(\mathrm{~s}, 1 \mathrm{H}, \mathrm{D}_{2} \mathrm{O}\right.$ exchangeable, $\mathrm{NH}$ amide), 8.98-9.24 (m, $2 \mathrm{H}, \mathrm{Ar}-\mathrm{H}), 11.29$ ( $\mathrm{s}, 1 \mathrm{H}, \mathrm{D}_{2} \mathrm{O}$ exchangeable, $\mathrm{NH}$ isatin), 12.52 ( $\mathrm{s}, 1 \mathrm{H}, \mathrm{D}_{2} \mathrm{O}$ exchangeable, $\mathrm{NH}$ hydrazide), 13.57 (s, $1 \mathrm{H}, \mathrm{D}_{2} \mathrm{O}$ exchangeable, $\mathrm{NH}$ hydrazide); HR-MS: 379.9433. Anal. Calcd. for $\mathrm{C}_{20} \mathrm{H}_{21} \mathrm{~N}_{5} \mathrm{O}_{3}$ (379.41): $\mathrm{C}$ (63.31), $\mathrm{H}(5.58), \mathrm{N}$ (18.46). Found: C (63.30), H (5.59), N (18.49).

N-(1-(2-(5-Bromo-2-oxoindolin-3-ylidene)hydrazinyl)-4-meth$y l-1$-oxopentan-2-yl)nicotinamide $(6 c)$. Yield (88\%); m. p. $178-180^{\circ} \mathrm{C} ;-[\alpha]_{\mathrm{D}}^{25}=-120(c=0.01, \mathrm{MeOH}) ; \mathrm{IR}(\mathrm{KBr})$ $\nu_{\max } / \mathrm{cm}^{-1} 3413-3233(\mathrm{NH}), 1701(\mathrm{C}=\mathrm{O}), 1639(\mathrm{C}=\mathrm{N}) ;{ }^{1} \mathrm{H}$ NMR $\left(\mathrm{DMSO}-d_{6}\right) \delta 0.93-1.10\left(\mathrm{~m}, 6 \mathrm{H}, 2 \mathrm{CH}_{3}\right), 1.63-1.92(\mathrm{~m}$, 
$\left.3 \mathrm{H},-\mathrm{C} \underline{\mathrm{H}}\left(\mathrm{CH}_{3}\right)_{2}+-\mathrm{CH}_{2}\right), 4.66-4.67(\mathrm{~m}, 1 \mathrm{H},-\mathrm{NH}-\mathrm{C} \underline{\mathrm{H}}-\mathrm{CO})$, 5.54-5.55 (m, 1H, -NH-CH-CO), 6.94-7.56 (m, 4H, Ar-H), $8.29(\mathrm{~s}, 1 \mathrm{H}, \mathrm{Ar}-\mathrm{H}), 8.76\left(\mathrm{~s}, \overline{\mathrm{H}}, \mathrm{D}_{2} \mathrm{O}\right.$ exchangeable, $\mathrm{NH}$ amide), 9.00-9.26 (m, 2H, Ar- $\mathrm{H}), 11.41$ ( $\mathrm{s}, 1 \mathrm{H}, \mathrm{D}_{2} \mathrm{O}$ exchangeable, $\mathrm{NH}$ isatin), 12.45 ( $\mathrm{s}, 1 \mathrm{H}, \mathrm{D}_{2} \mathrm{O}$ exchangeable, $\mathrm{NH}$ hydrazide), 13.52 (s, $1 \mathrm{H}, \mathrm{D}_{2} \mathrm{O}$ exchangeable, $\mathrm{NH}$ hydrazide); HR-MS: 458.1546. Anal. Calcd. for $\mathrm{C}_{20} \mathrm{H}_{20} \mathrm{BrN}_{5} \mathrm{O}_{3}$ (458.31): $\mathrm{C}$ (52.41), $\mathrm{H}$ (4.40), $\mathrm{N}$ (15.28). Found: C (52.39), H (4.43), N (15.26).

N-(1-(2-(5-Chloro-2-oxoindolin-3-ylidene)hydrazinyl)-4-methyl-1-oxopentan-2-yl)nicotinamide (6d). Yield (90\%); m. p. $194-196^{\circ} \mathrm{C} ;-[\alpha]_{\mathrm{D}}^{25}=-70(c=0.01, \mathrm{MeOH}) ; \mathrm{IR}(\mathrm{KBr})$ $v_{\max } / \mathrm{cm}^{-1} 3413-3232(\mathrm{NH}), 1700(\mathrm{C}=\mathrm{O}), 1641(\mathrm{C}=\mathrm{N}) ;{ }^{1} \mathrm{H}$ NMR (DMSO- $\left.d_{6}\right) \delta 0.93-1.10\left(\mathrm{~m}, 6 \mathrm{H}, 2 \mathrm{CH}_{3}\right), 1.62-1.92(\mathrm{~m}$, $\left.3 \mathrm{H},-\mathrm{CH}\left(\mathrm{CH}_{3}\right)_{2}+-\mathrm{CH}_{2}\right), 4.66-4.67(\mathrm{~m}, 1 \mathrm{H},-\mathrm{NH}-\mathrm{C}-\mathrm{H}-\mathrm{CO})$, 5.54-4.55 (m, 1H, -NH-CH-CO), 6.90-7.68 (m, 4H, Ar-H), $8.30(\mathrm{~s}, 1 \mathrm{H}, \mathrm{Ar}-\mathrm{H}), 8.78$ (s, $1 \mathrm{H}, \mathrm{D}_{2} \mathrm{O}$ exchangeable, $\mathrm{NH}$ amide), 9.00-9.26 (m, 2H, Ar-H), $11.42\left(\mathrm{~s}, 1 \mathrm{H}, \mathrm{D}_{2} \mathrm{O}\right.$ exchangeable, $\mathrm{NH}$ isatin), 12.43 (s, $1 \mathrm{H}, \mathrm{D}_{2} \mathrm{O}$ exchangeable, $\mathrm{NH}$ hydrazide), 13.51 (s, $1 \mathrm{H}, \mathrm{D}_{2} \mathrm{O}$ exchangeable, $\mathrm{NH}$ hydrazide); HR-MS: 413.0183. Anal. Calcd. for $\mathrm{C}_{20} \mathrm{H}_{20} \mathrm{ClN}_{5} \mathrm{O}_{3}$ (413.86): C (58.04), H (4.87), N (16.92). Found: C (58.01), H (4.83), N (16.95).

N-(1-Oxo-1-(2-(2-oxoindolin-3-ylidene)hydrazinyl)-3-phenylpropan-2-yl)nicotinamide (6e). Yield (84\%); m. p. $218-220^{\circ} \mathrm{C}$; $-[\alpha]_{\mathrm{D}}^{25}=-110(c=0.03, \mathrm{MeOH}) ; \mathrm{IR}(\mathrm{KBr}) v_{\max } / \mathrm{cm}^{-1} 3413-$ $3228(\mathrm{NH}), 1702(\mathrm{C}=\mathrm{O}), 1621(\mathrm{C}=\mathrm{N}) ;{ }^{1} \mathrm{H}$ NMR $\left(\right.$ DMSO- $\left.d_{6}\right)$ $\delta 3.08-3.33\left(\mathrm{~m}, 2 \mathrm{H},-\mathrm{CH}_{2}\right), 4.90-4.91(\mathrm{~m}, 1 \mathrm{H},-\mathrm{NH}-\mathrm{C} \underline{\mathrm{H}}-\mathrm{CO})$, 5.67-4.68 (m, 1H, -NH-C $\underline{H}-\mathrm{CO}), 7.11-7.58(\mathrm{~m}, 10 \mathrm{H}, \mathrm{Ar}-\mathrm{H})$, 8.19 (s, $1 \mathrm{H}, \mathrm{Ar}-\mathrm{H}), 8.72\left(\mathrm{~s}, 1 \overline{\mathrm{H}}, \mathrm{D}_{2} \mathrm{O}\right.$ exchangeable, $\mathrm{NH}$ amide), 9.01-9.60 (m, 2H, Ar-H), 11.35 (s, $1 \mathrm{H}, \mathrm{D}_{2} \mathrm{O}$ exchangeable, $\mathrm{NH}$ isatin), 12.61 ( $\mathrm{s}, 1 \mathrm{H}, \mathrm{D}_{2} \mathrm{O}$ exchangeable, $\mathrm{NH}$ hydrazide), 13.55 (s, $1 \mathrm{H}, \mathrm{D}_{2} \mathrm{O}$ exchangeable, NH hydrazide); HR-MS: 413.1841. Anal. Calcd. for $\mathrm{C}_{16} \mathrm{H}_{15} \mathrm{~N}_{5}$ OS (413.43): C (66.82), H (4.63), $\mathrm{N}$ (16.96). Found: C (66.78), H (4.61), N (16.99).

$\mathrm{N}$-(1-(2-(5-Bromo-2-oxoindolin-3-ylidene)hydrazinyl)-1-oxo3-phenylpropan-2-yl)nicotinamide (6f). Yield (81\%); m. p. $200-204^{\circ} \mathrm{C} ;-[\alpha]_{\mathrm{D}}^{25}=-105(c=0.01, \mathrm{MeOH}) ; \mathrm{IR}(\mathrm{KBr})$ $v_{\max } / \mathrm{cm}^{-1} 3410-3230(\mathrm{NH}), 1700(\mathrm{C}=\mathrm{O}), 1630(\mathrm{C}=\mathrm{N}) ;{ }^{1} \mathrm{H}$ NMR (DMSO- $\left.d_{6}\right) \delta 3.07-3.39\left(\mathrm{~m}, 2 \mathrm{H},-\mathrm{CH}_{2}\right), 4.90-4.91$ (m, 1H, -NH-CH-CO), 5.64-4.65 (m, $1 \mathrm{H},-\mathrm{NH}-\mathrm{CH}-\mathrm{CO})$, 6.86-7.96 (m, 9) $\mathrm{H}, \operatorname{Ar}-\mathrm{H}), 8.17$ (s, $1 \mathrm{H}, \operatorname{Ar}-\mathrm{H}), 8.76(\mathrm{~s}, 1 \mathrm{H}$, $\mathrm{D}_{2} \mathrm{O}$ exchangeable, $\mathrm{NH}$ amide), 9.04-9.41 (m, $\left.2 \mathrm{H}, \mathrm{Ar}-\mathrm{H}\right)$, $11.48\left(\mathrm{~s}, 1 \mathrm{H}, \mathrm{D}_{2} \mathrm{O}\right.$ exchangeable, $\mathrm{NH}$ isatin), $12.47(\mathrm{~s}, 1 \mathrm{H}$, $\mathrm{D}_{2} \mathrm{O}$ exchangeable, $\mathrm{NH}$ hydrazide), $13.48\left(\mathrm{~s}, 1 \mathrm{H}, \mathrm{D}_{2} \mathrm{O}\right.$ exchangeable, NH hydrazide); HR-MS: 491.0701. Anal. Calcd. for $\mathrm{C}_{16} \mathrm{H}_{15} \mathrm{~N}_{5}$ OS (492.32): C (56.11), $\mathrm{H}$ (3.69), $\mathrm{N}$ (14.23). Found: C (56.10), H (3.65), N (14.26).

N-(1-(2-(5-Chloro-2-oxoindolin-3-ylidene)hydrazinyl)-1-oxo3-phenylpropan-2-yl)nicotinamide (6g). Yield (80\%); m. p. $226-230^{\circ} \mathrm{C} ;-[\alpha]_{\mathrm{D}}^{25}=-20(c=0.01, \mathrm{MeOH}) ; \mathrm{IR}(\mathrm{KBr})$ $\nu_{\max } / \mathrm{cm}^{-1} 3413-3280(\mathrm{NH}), 1741-1692(\mathrm{C}=\mathrm{O}), 1620(\mathrm{C}=\mathrm{N})$; ${ }^{1} \mathrm{H}$ NMR (DMSO- $\left.d_{6}\right) \delta 3.07-3.39\left(\mathrm{~m}, 2 \mathrm{H},-\mathrm{CH}_{2}\right), 4.90-4.91$ (m, $1 \mathrm{H},-\mathrm{NH}-\mathrm{C} \underline{H}-\mathrm{CO}), 5.64-4.65(\mathrm{~m}, 1 \mathrm{H},-\mathrm{NH}-\mathrm{C} \underline{H}-\mathrm{CO})$, 6.93-7.54 (m, 9 $\overline{\mathrm{H}}, \mathrm{Ar}-\mathrm{H}), 8.18(\mathrm{~s}, 1 \mathrm{H}, \operatorname{Ar}-\mathrm{H}), 8.73(\mathrm{~s}, 1 \mathrm{H}$, $\mathrm{D}_{2} \mathrm{O}$ exchangeable, $\mathrm{NH}$ amide), 9.0-9.40 (m, 2H, Ar-H), $10.97\left(\mathrm{~s}, 1 \mathrm{H}, \mathrm{D}_{2} \mathrm{O}\right.$ exchangeable, $\mathrm{NH}$ isatin), $11.40(\mathrm{~s}, 1 \mathrm{H}$, $\mathrm{D}_{2} \mathrm{O}$ exchangeable, $\mathrm{NH}$ hydrazide), $11.70\left(\mathrm{~s}, 1 \mathrm{H}, \mathrm{D}_{2} \mathrm{O}\right.$ exchangeable, $\mathrm{NH}$ hydrazide); HR-MS: 447.4478. Anal. Calcd. for $\mathrm{C}_{23} \mathrm{H}_{18} \mathrm{ClN}_{5} \mathrm{O}_{3}$ (447.87): $\mathrm{C}$ (61.68), $\mathrm{H}$ (4.05), $\mathrm{N}$ (15.64). Found: C (61.69), H (4.02), N (15.67).

\subsection{Biological Study}

2.2.1. Samples Preparation. All samples were dissolved in dimethyl sulfoxide (RFCL Limited, New Delhi, India) (DMSO) at $10 \mathrm{mg} / \mathrm{mL}$ concentration, in comparison with different standard antibiotics as shown in Table 1. Antibiotic discs of streptomycin (S) (10 $\mu \mathrm{g})$ and tetracycline (TE) (30 $\mu \mathrm{g})$ were used as positive control for bacteria, neomycin $(\mathrm{N})$ $(30 \mu \mathrm{g})$ and nystatin (NY) $(100 \mu \mathrm{g})$ were used for fungi, and sterilized paper discs without compounds or antibiotics were used as negative controls for both bacteria and fungi. The experiment was performed in triplicate.

2.2.2. Antimicrobial Activity. The ability to inhibit the growth of Gram-positive and Gram-negative bacteria, yeasts, and filamentous fungi was observed using an overlay method [29].

\subsubsection{Antimicrobial Assay}

(1) Strains Used. The common pathogenic and food spoilage microorganisms were selected for their relevance in bakery products and other foods: the Gram-positive bacteria were Bacillus subtilis and Staphylococcus aureus, Gram-negative bacteria was Escherichia coli, yeasts such as Candida albicans, and fungi was Aspergillus niger.

(2) Media Used. The bacteria were slanted on nutrient agar (Merck, Darmstadt, Germany), yeast was slanted and mentioned onSabaroud's agar medium (Lab M., Bury, Lancashire, UK), and the fungi were slanted and mentioned on the Potato Dextrose Agar medium (Lab M Limited, Bury, Lancashire, UK). Mueller-Hinton agar (Lab M., Bury, Lancashire, UK) following the manufacturer's instructions was used for the assay.

(3) Bioassay. The antibacterial screening was performed by the well diffusion agar method described by Moosdeen et al. [30]. The organisms were streaked in radial patterns on the agar plates. Plates were incubated under aerobic conditions at $37^{\circ} \mathrm{C}$ and $28^{\circ} \mathrm{C}$ for $24 \mathrm{~h}$ and $48 \mathrm{~h}$ for bacteria and fungi, respectively. In order to obtain comparable results, all prepared solutions were treated under the same conditions. All tests were performed for three replicates. Plates were examined for evidence of antimicrobial activities, represented by a zone of inhibition of microorganism's growth around the holes, and diameters of clear zones were expressed in millimeters [31].

(4) Determination of Minimum Inhibitory Concentration (MIC). The in vitro minimum inhibitory concentration (MIC) of the synthesized compounds was determined by agar well diffusion method. Nutrient broth medium was used to 
TABLE 1: Antimicrobial activity of the synthesized compounds at $10 \mathrm{mg} / \mathrm{mL}$.

\begin{tabular}{|c|c|c|c|c|c|}
\hline \multirow{3}{*}{ Comp. number } & \multicolumn{3}{|c|}{ Bacteria } & \multicolumn{2}{|c|}{ Fungi } \\
\hline & \multicolumn{2}{|c|}{ Gram-positive } & \multirow{2}{*}{$\begin{array}{c}\text { Gram-negative } \\
\text { E. coli } \\
\end{array}$} & \multirow{2}{*}{$\begin{array}{l}\text { Unicellular } \\
\text { C. albicans }\end{array}$} & \multirow{2}{*}{$\begin{array}{c}\text { Filamentous } \\
\text { Aspergillus niger }\end{array}$} \\
\hline & B. subtilis & S. aureus & & & \\
\hline $4 b$ & $15.13 \pm 0.1768$ & $00.00 \pm 0.0000$ & $00.00 \pm 0.0000$ & $11.125 \pm 0.1768$ & $00.00 \pm 0.0000$ \\
\hline $6 a$ & $30.18 \pm 0.2475$ & $22.12 \pm 0.1626$ & $25.125 \pm 0.1768$ & $30.125 \pm 0.1768$ & $00.00 \pm 0.0000$ \\
\hline $6 b$ & $18.17 \pm 0.2404$ & $15.10 \pm 0.1414$ & $15.125 \pm 0.1768$ & $15.10 \pm 0.1414$ & $15.16 \pm 0.2263$ \\
\hline $6 c$ & $23.18 \pm 0.2475$ & $23.18 \pm 0.2475$ & $20.175 \pm 0.2475$ & $20.115 \pm 0.1626$ & $00.00 \pm 0.1768$ \\
\hline $6 d$ & $20.10 \pm 0.1414$ & $24.08 \pm 0.1060$ & $20.175 \pm 0.2475$ & $20.17 \pm 0.2404$ & $00.00 \pm 0.0000$ \\
\hline $6 e$ & $25.20 \pm 0.2828$ & $30.13 \pm 0.1768$ & $30.15 \pm 0.2121$ & $25.175 \pm 0.2475$ & $00.00 \pm 0.0000$ \\
\hline $6 f$ & $27.18 \pm 0.2475$ & $32.10 \pm 0.1410$ & $32.13 \pm 0.1768$ & $25.10 \pm 0.1414$ & $00.00 \pm 0.0000$ \\
\hline $6 \mathrm{~g}$ & $15.13 \pm 0.176$ & $15.00 \pm 0.0000$ & $15.10 \pm 0.1414$ & $00.00 \pm 0.0000$ & $14.10 \pm 0.1414$ \\
\hline $\mathrm{S}^{*}=10 \mu \mathrm{g}$ & $14.00 \pm 0.0000$ & $00.00 \pm 0.0000$ & $12.00 \pm 0.0000$ & $00.00 \pm 0.0000$ & $00.00 \pm 0.0000$ \\
\hline $\mathrm{TE}^{*}=30 \mu \mathrm{g}$ & $18.00 \pm 0.0000$ & $00.00 \pm 0.0000$ & $23.5 \pm 2.1213$ & $00.00 \pm 0.0000$ & $00.00 \pm 0.0000$ \\
\hline $\mathrm{N}^{*}=30 \mu \mathrm{g}$ & $00.00 \pm 0.0000$ & $00.00 \pm 0.0000$ & $00.00 \pm 0.0000$ & $16.00 \pm 1.4142$ & $15.00 \pm 0.0000$ \\
\hline $\mathrm{NS}^{*}=100 \mu \mathrm{g}$ & $00.00 \pm 0.0000$ & $00.00 \pm 0.0000$ & $00.00 \pm 0.0000$ & $00.00 \pm 0.0000$ & $15.00 \pm 0.0000$ \\
\hline
\end{tabular}

${ }^{*}$ S: streptomycin; TE: tetracycline; N: neomycin, and NS: nystatin.

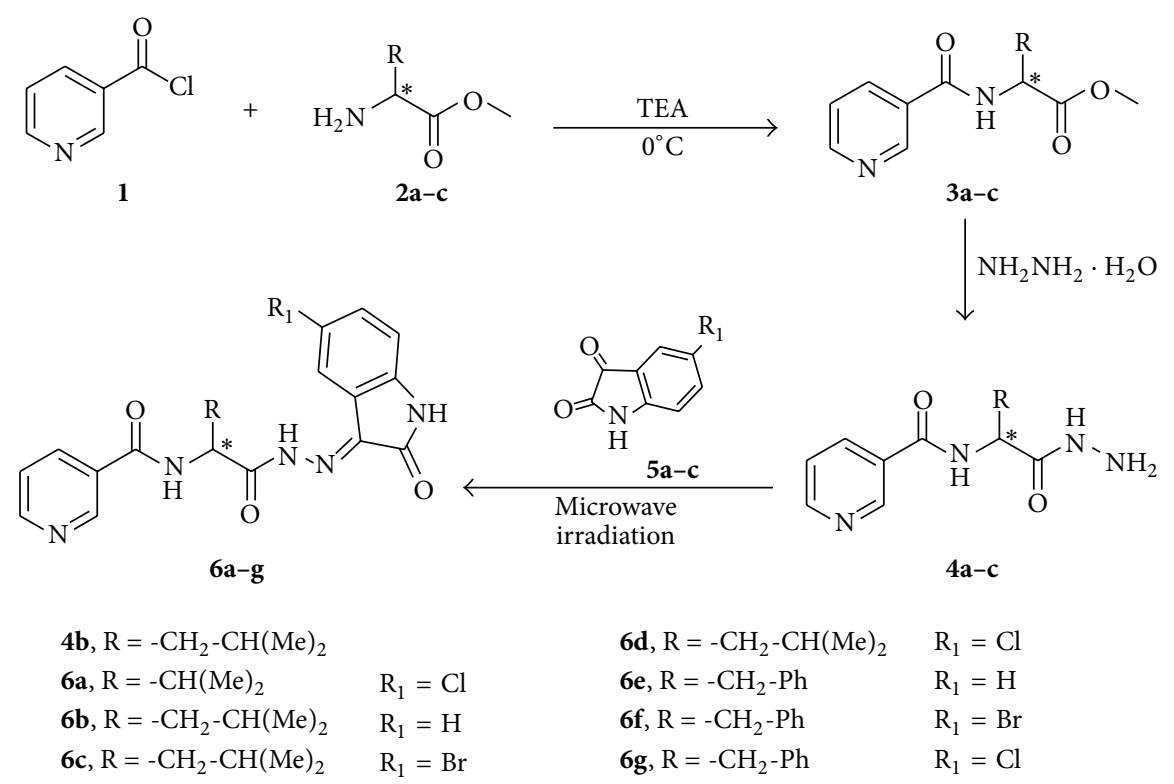

Scheme 1: Synthetic routes for compounds $4 \mathbf{b}$ and $\mathbf{6 a}-\mathbf{g}$.

prepare different concentrations ranging from $50 \mu \mathrm{g} / \mathrm{mL}$ to $500 \mu \mathrm{g} / \mathrm{mL}$ by serial dilutions. Each prepared concentration in tubes was inoculated with $100 \mu \mathrm{L}$ of each of the $10^{6} \mathrm{cfu} / \mathrm{mL}$ bacterial and fungal strains and the assay was applied by agar well diffusion method. Blank nutrient broth was used as negative control. The plates were incubated aerobically at $37^{\circ} \mathrm{C}$ for $18 \mathrm{~h}$ to $24 \mathrm{~h}$ for bacterial strains and $25^{\circ} \mathrm{C}$ for $48 \mathrm{~h}$ for fungal strains. The lowest inhibition zone in the series dilution was taken as the MIC.

\section{Results and Discussion}

3.1. Chemistry. In the present work, we suggested the coupling of two heterocyclic compounds via certain amino acid as a bridge and studied the biological activities of these compounds as potent antimicrobial agents. The preparation and characterization of new compounds represented by the $\mathbf{4 b}$ and $\mathbf{6} \mathbf{a}-\mathbf{g}$, as well as the acid chloride method of coupling, was successfully applied as the method of choice for assembling the peptide bond. Herein, a series of chiral linear carboxamide derivatives incorporating peptide linkage have been prepared via the coupling of nicotinoyl chloride $\mathbf{1}$ with suited L-amino acid methyl esters $\mathbf{2 a - c}$ including valine, leucine, and phenylalanine which produced the corresponding peptide methyl esters $3 \mathbf{a}-\mathbf{c}$, respectively. Hydrazinolysis of esters 3a-c with hydrazine hydrate 99\% produced the hydrazide derivatives $\mathbf{4 a - c}$, respectively. The latter compounds were acidified and coupled with isatin derivatives $5 \mathbf{a}-\mathbf{c}$ in ethanol and irradiated successfully under microwave irradiation at 420 watts $\left(\sim 110^{\circ} \mathrm{C}\right)$ for $5-10$ minutes to afford the corresponding Schiff bases $6 \mathbf{a}-\mathbf{g}$, respectively; see Scheme 1. The results indicated that microwave technique 
TABLE 2: MIC of different compounds against most of tested pathogens.

\begin{tabular}{lcccccccc}
\hline \multirow{2}{*}{ Comp. number } & \multicolumn{3}{c}{ Inhibition zone diameters $(\mathrm{mm})$} & \multicolumn{3}{c}{$\mathrm{MIC}(\mu \mathrm{g} / \mathrm{mL})$} \\
& B. subtilis & E. coli & S. aureus & C. albicans & B. subtilis & E. coli & S. aureus & C. albicans \\
\hline 6e & 11 & 11 & 11 & 11 & 50 & 50 & 50 & 50 \\
6f & 11 & 12 & 12 & 12 & 100 & 50 & 50 & 50 \\
6a & 11 & 11 & 11 & 12 & 500 & 75 & 500 & 500 \\
6c & 11 & 11 & 11 & - & 50 & 75 & - \\
6d & 11 & 11 & - & - & 75 & 50 & - \\
\hline
\end{tabular}

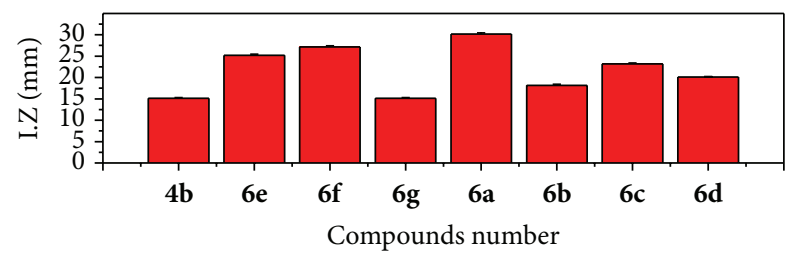

B. subtilis

(a)

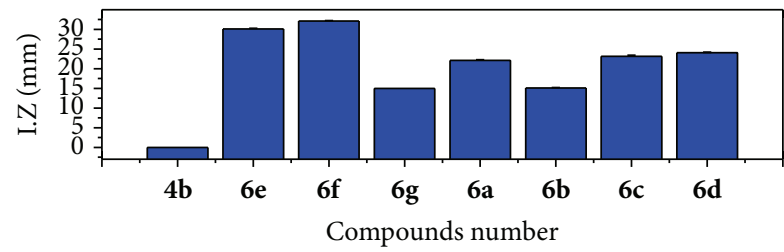

S. aureus

(b)

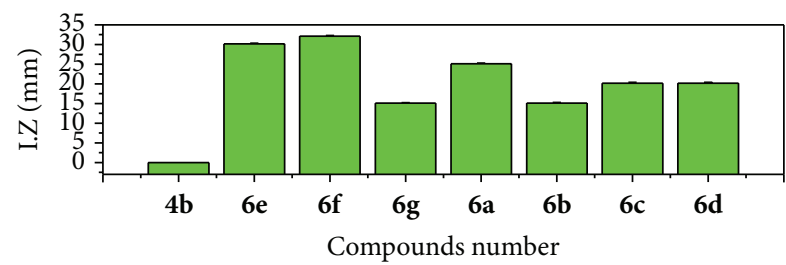

E. coli

(c)

FIGURE 1: Antibacterial activity of the synthesized compounds at concentration of $10 \mathrm{mg} / \mathrm{mL}$ : (a) B. subtilis, (b) S. aureus and (c) E. coli.

gave improved yield in less reaction time. So this confirms that the microwave (MW) synthesis has been shown to be an invaluable tool for medicinal chemistry and drug discovery applications since it often dramatically reduces reaction times, typically from days or hours to minutes or even seconds [32].

3.2. Biological Evaluations. The results in Table 1 showed that most of the compounds have a strong antibacterial inhibitory effect against most of tested pathogens. Most of the compounds showed a strong inhibitory effect against Grampositive bacteria such as Bacillus subtilis and S. aureus except compound $\mathbf{4 b}$, which showed a negative inhibitory effect in comparison with the antibacterial standard streptomycin. The inhibitory zone diameters ranged from $15 \mathrm{~mm}$ to $30 \mathrm{~mm}$ and were illustrated in Figures 1(a) and 1(b). A Gram-positive

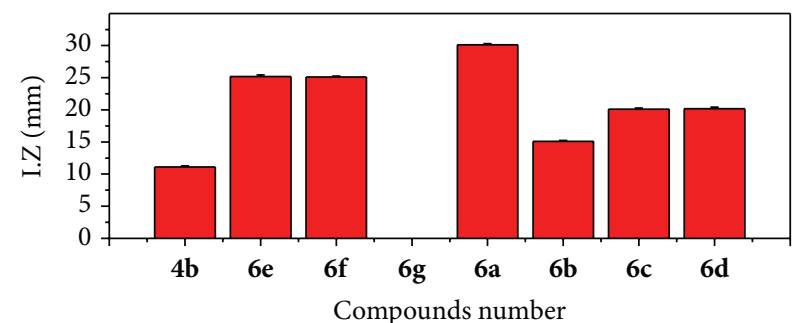

C. albicans

(a)

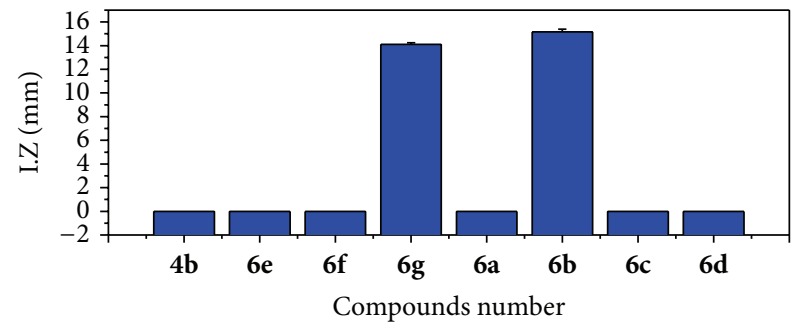

A. niger

(b)

FIGURE 2: Antifungal activity of the synthesized compounds at concentration of $10 \mathrm{mg} / \mathrm{mL}$ : (a) C. albicans and (b) A. niger.

bacterium infects the upper respiratory tract. So, these compounds can be used in the treatment of these pathogen groups. Also, a strong inhibitory effect ( $15 \mathrm{~mm}$ to $32 \mathrm{~mm}$ ) was noticed against Escherichia coli as example of Gram-negative bacteria except compound $\mathbf{4} \mathbf{b}$ which showed a negative inhibitory effect, illustrated in Figure 1(c).

The antifungal activities are presented in Figure 2, in case of unicellular fungi. Compounds $4 b, 6 e, 6 f, 6 a, 6 b, 6 c$, and $\mathbf{6 d}$ showed a strong antifungal effect against Candida albicans, while compound $\mathbf{6 g}$ was characterized by negative antifungal effect; see Figure 2(a). In case of filamentous fungi, compounds $\mathbf{6 b}$ and $\mathbf{6} \mathbf{g}$ have the significant antifungal effect against Aspergillus niger while the other compounds did not show any inhibitory effect, compared to the antifungal standard,neomycin and nystatin (Figure 2(b)).

Aspergillus niger is one of the fungal pathogens that can infect the respiratory tract. A. niger is a causative agent of pulmonary diseases including aspergillosis, bronchial asthma, and acute allergic alveolitis. The fungus colonizes old tuberculosis or bronchiectatic cavities, in which it forms a large colony (aspergilloma); or it may actually invade the lung 


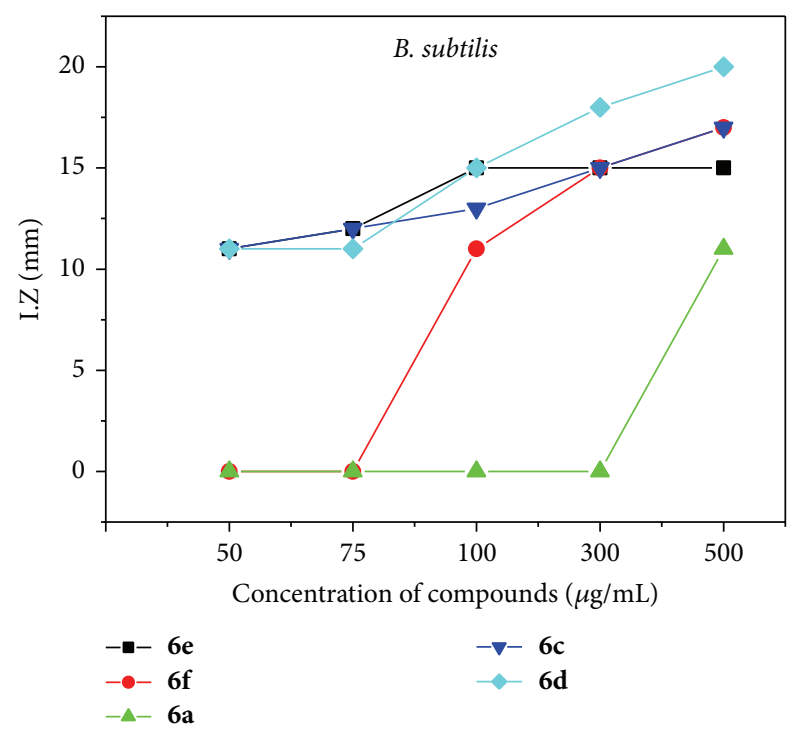

(a)

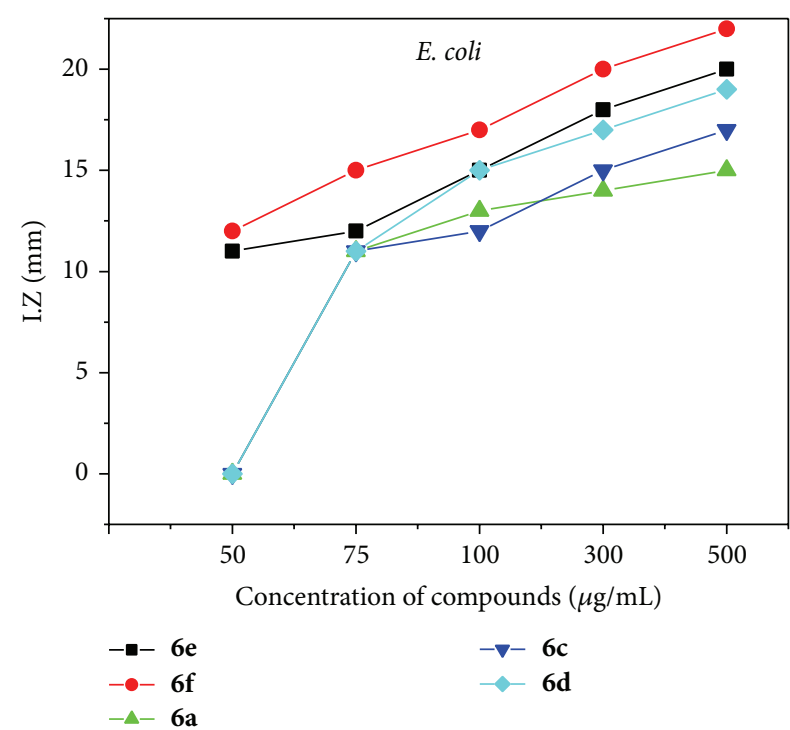

(c)

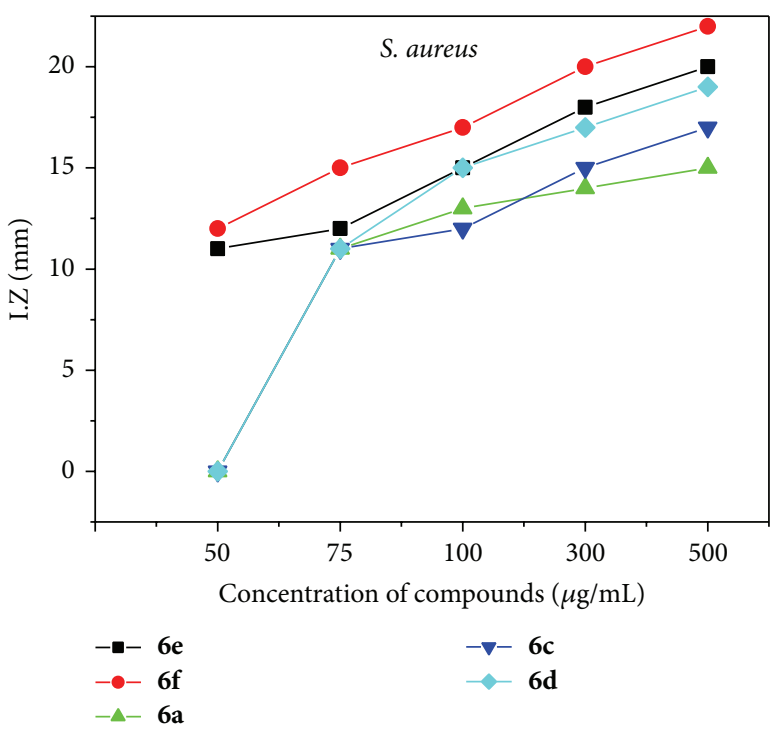

(b)

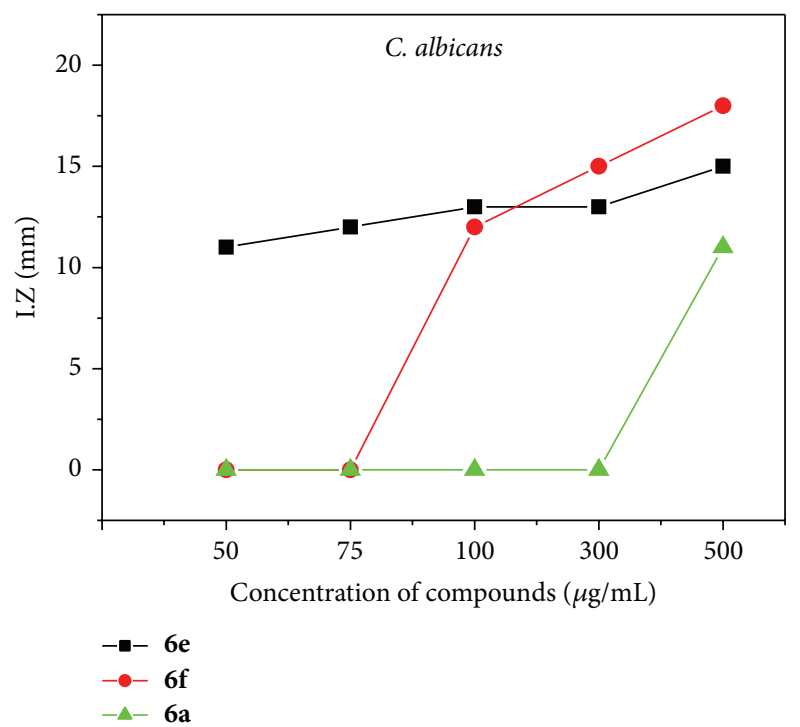

(d)

Figure 3: MIC of compounds against Gram-positive bacteria (B. subtilis) (a) and (S. aureus) (b), Gram-negative bacteria (E. coli) (c), and yeast (C. albicans) (d).

tissue to produce hemorrhagic and necrotizing pneumonia [33]. The minimum inhibitory concentrations (MIC) of the synthesized compounds are presented in Table 2 and Figures 3(a), 3(b), 3(c), and 3(d). The MIC was in the range from $50 \mu \mathrm{g} / \mathrm{mL}$ to $500 \mu \mathrm{g} / \mathrm{mL}$ based on the compounds tested. The MIC of compound $6 \mathrm{e}$ was $50 \mu \mathrm{g} / \mathrm{mL}$ against all pathogens tested. The MIC of $\mathbf{6} \mathbf{f}$ was $50 \mu \mathrm{g} / \mathrm{mL}$ against all strains except Bacillus subtilis which displayed MIC of $100 \mu \mathrm{g} / \mathrm{mL}$. On the other hand, the MIC of $\mathbf{6 c}$ was $50 \mu \mathrm{g} / \mathrm{mL}$ against Bacillus subtilis.

3.3. Structure Activity Relationship (SAR). The antimicrobial activity of compounds $\mathbf{6 a}, \mathbf{6 c}, \mathbf{6 d}, \mathbf{6 e}$, and $\mathbf{6}$ f demonstrated significant activities against all tested strains. The MIC was found in the following order: $\mathbf{6 e}>\mathbf{6 f}>\mathbf{6 c}>\mathbf{6 d}>\mathbf{6 a}$. Compound 6e having unsubstituted isatin and phenylalanine as bridge was found to be the most active compound against all strains with MIC of $50 \mu \mathrm{g} / \mathrm{mL}$. Compound $\mathbf{6 f}$ having 5-bromoisatin and phenylalanine as bridge was found to have significant activity. From the antimicrobial activity data, structure activity relationship can be concluded as follows.

(1) The compounds with phenylalanine as a bridge weremore active than leucine and valine as a bridge.

(2) The compound with unsubstituted isatin and phenylalanine as bridge was the most active compound.

(3) The compound with 5-bromoisatin and phenylalanine as bridge displayed significant activity. 
(4) The compound with 5-chloroisatin and valine as bridge displayed the least activity.

(5) The compounds with 5-chloro/5-bromoisatin and leucine as bridge displayed the moderate activity.

\section{Conclusion}

The coupling reaction of nicotinic acid with certain L-amino acid methyl ester was performed by the use of acid chloride method. The ester obtained was reacted with hydrazine hydrate $99 \%$ to give the corresponding hydrazide which was reacted with isatin and substituted isatin under the application of microwave irradiation to produce final compounds $(6 \mathbf{a}-\mathbf{g})$. The synthesized compounds were characterized by spectroscopic data and checked for purity. The in vitro antimicrobial activity of the synthesized compounds was performed against Gram-positive bacteria (Bacillus subtilis and S. aureus), Gram-negative bacteria (Escherichia coli), yeast (Candida albicans), and filamentous fungi (Aspergillus niger). Most of the samples displayed significant antimicrobial activity. Compound 6 e having unsubstituted isatin and phenylalanine as bridge was found to be the most active compound against all strains with MIC of $50 \mu \mathrm{g} / \mathrm{mL}$. Compound $\mathbf{6 e}$ can be used as a lead compound for further development of more potent and broad spectrum antimicrobial agent.

\section{Conflict of Interests}

The authors declare that there is no conflict of interests regarding the publication of this paper.

\section{Acknowledgment}

The authors extend their appreciation to the Deanship of Scientific Research at King Saud University for funding the work through the Research Group no. RG 1435-006.

\section{References}

[1] "Drugs and Suplementes, Niacin (vitamin B3, nicotinic acid) Niacinamide," http://www.mayoclinic.org/drugs-supplements/ niacin--niacinamide\%20/background/hrb-20059838.

[2] M. C. S. Lourenço, M. V. N. de Souza, A. C. Pinheiro et al., "Evaluation of anti-tubercular activity of nicotinic and isoniazid analogues," Arkivoc, vol. 2007, no. 15, pp. 181-191, 2007.

[3] G. J. Hageman and R. H. Stierum, "Niacin, poly(ADP-ribose) polymerase-1 and genomic stability," Mutation Research: Fundamental and Molecular Mechanisms of Mutagenesis, vol. 475, no. 1-2, pp. 45-56, 2001.

[4] L. A. Carlson, "Nicotinic acid: the broad-spectrum lipid drug. A 50th anniversary review," Journal of Internal Medicine, vol. 258, no. 2, pp. 94-114, 2005.

[5] Q. Lin, D. Fang, X. Hou et al., "HCV peptide (C5A), an amphipathic $\alpha$-helical peptide of hepatitis virus $\mathrm{C}$, is an activator of $\mathrm{N}$-formyl peptide receptor in human phagocytes," The Journal of Immunology, vol. 186, no. 4, pp. 2087-2094, 2011.

[6] P. Ruchala, M. Navab, C.-L. Jung et al., "Oxpholipin 11D: an anti-inflammatory peptide that binds cholesterol and oxidized phospholipids," PLoS ONE, vol. 5, no. 4, Article ID e10181, 2010.
[7] F. Chen, F. Zhang, A. Wang et al., "Recent progress in the chemo-enzymatic peptide synthesis," African Journal of Pharmacy and Pharmacology, vol. 4, no. 10, pp. 721-730, 2010.

[8] L. L. Burrows, M. Stark, C. Chan, E. Glukhov, S. Sinnadurai, and C. M. Deber, "Activity of novel non-amphipathic cationic antimicrobial peptides against Candida species," Journal of Antimicrobial Chemotherapy, vol. 57, no. 5, pp. 899-907, 2006.

[9] V. Krishnakumari, S. Singh, and R. Nagaraj, "Antibacterial activities of synthetic peptides corresponding to the carboxyterminal region of human $\beta$-defensins 1-3," Peptides, vol. 27, no. 11, pp. 2607-2613, 2006.

[10] B. Bacsa, B. Desai, G. Dibó, and O. Kappe, "Rapid solid-phase peptide synthesis using thermal and controlled microwave irradiation," Journal of Peptide Science, vol. 12, no. 10, pp. 633638, 2006.

[11] K. J. Jensen and J. Brask, "Carbohydrates in peptide and protein design,” Biopolymers, vol. 80, no. 6, pp. 747-761, 2005.

[12] S. Abdel Rahman, A. El-Kafrawy, A. Hattaba, and M. F. Anwer, "Optimization of solid-phase synthesis of difficult peptide sequences via comparison between different improved approaches," Amino Acids, vol. 33, no. 3, pp. 531-536, 2007.

[13] J. K. Murray and S. H. Gellman, "Microwave-assisted parallel synthesis of a 14-helical $\beta$-peptide library," Journal of Combinatorial Chemistry, vol. 8, no. 1, pp. 58-67, 2006.

[14] T. Matsushita, H. Hinou, M. Fumoto et al., "Construction of highly glycosylated mucin-type glycopeptides based on microwave-assisted solid-phase syntheses and enzymatic modifications," Journal of Organic Chemistry, vol. 71, no. 8, pp. 30513063, 2006.

[15] S. N. Pandeya, S. Smitha, M. Jyoti, and S. K. Sridhar, "Biological activities of isatin and its derivatives," Acta Pharmaceutica, vol. 55, no. 1, pp. 27-46, 2005.

[16] W. Chu, J. Zhang, C. Zeng et al., "N-benzylisatin sulfonamide analogues as potent caspase- 3 inhibitors: synthesis, in vitro activity, and molecular modeling studies," Journal of Medicinal Chemistry, vol. 48, no. 24, pp. 7637-7647, 2005.

[17] W. Chu, J. Rothfuss, Y. Chu, D. Zhou, and R. H. Mach, "Synthesis and in vitro evaluation of sulfonamide isatin Michael acceptors as small molecule inhibitors of caspase-6," Journal of Medicinal Chemistry, vol. 52, no. 8, pp. 2188-2191, 2009.

[18] Z. H. Chohan, H. Pervez, A. Rauf, K. M. Khan, and C. T. Supuran, "Isatin-derived antibacterial and antifungal compounds and their transition metal complexes," Journal of Enzyme Inhibition and Medicinal Chemistry, vol. 19, no. 5, pp. 417-423, 2004.

[19] B. R. Nathani, K. S. Pandya, M. M. Jeni, and M. R. Patel, "Synthesis and antimicrobial activity of some new isatins derivatives," Der Pharma Chemica, vol. 3, no. 4, pp. 367-372, 2011.

[20] T. Aboul-Fadl, H. A. Abdel-Aziz, M. K. Abdel-Hamid, T. Elsaman, J. Thanassi, and M. J. Pucci, "Schiff bases of indoline2,3-dione: potential novel inhibitors of mycobacterium tuberculosis (Mtb) DNA gyrase," Molecules, vol. 16, no. 9, pp. 78647879, 2011.

[21] A. M. Naglah, N. M. Khalifa, M. A. AL-Omar, H. M. Awad, and A. E. Amr, "In vitro antimicrobial activity of some newly synthesized polypeptide candidates," Digest Journal of Nanomaterials and Biostructures, vol. 9, no. 1, pp. 433-442, 2014.

[22] M. H. Abo-Ghalia and A. E. Amr, "Synthesis and investigation of a new cyclo-( $\mathrm{N} \alpha$-dipicolinoyl) pentapeptide of a breast and CNS cytotoxic activity and an ionophoric specifity," Amino Acids, vol. 26, pp. 283-289, 2004. 
[23] N. M. Khalifa, A. M. Naglah, M. A. Al-Omar, M. A. Abo-Ghalia, and A. E.-G. E. Amr, "Synthesis and reactions of new chiral linear carboxamides with an incorporated peptide linkage using nalidixic acid and amino acids as starting materials," Zeitschrift fur Naturforschung Section B: Journal of Chemical Sciences, vol. 69, no. 3, pp. 351-361, 2014.

[24] N. M. Khalifa, A. M. Naglah, M. A. Al-Omar, and A. E. Amr, "Synthesis and reactions of new chiral linear dipeptide candidates using nalidixic acid as starting material," Zeitschrift für Naturforschung B, vol. 69, pp. 728-736, 2014.

[25] S. E. Abdel Rahman, A. M. Naglah, M. A. Al-Omar, A. Kalmouch, and R. A. Amin, "Haematological measurements for some new erythropoietin hormone analogues synthesized by use of a modified method," Research on Chemical Intermediates, vol. 40, no. 4, pp. 1691-1702, 2014.

[26] M. A. Bhat, M. A. Al-Omar, A. M. Naglah, M. M. Abdulla, and H. K. Fun, "Synthesis and antitumor activity of 4-cyclohexyl/aryl-5-(pyridin-4-yl)-2,4-dihydro-3H-1,2,4-triazole-3-thiones," Medicinal Chemistry Research, 2014.

[27] D. A. Rockcliffe and A. E. Martell, "Copper(I) and copper(II) dinuclear complexes of a macrocyclic ligand derived from the 2:2 condensation of pyridine-2,6-dicarboxaldehyde and 1,4,7triazaheptane," Journal of Molecular Catalysis A: Chemical, vol. 99, no. 2, pp. 87-99, 1995.

[28] M. H. Abo Ghalia, E. M. Salem, S. Shoeb, and H. Zedan, "Synthesis of some N-nicotinoyl amino acid derivatives with high antitubercular activity," Polish Journal of Chemistry, vol. 53, no. 11, pp. 2239-2250, 1979.

[29] S. T. Williams, M. Goodfellow, G. Alderson, E. M. H. Wellington, P. H. A. Sneath, and M. J. Sackin, "Numerical classification of Streptomyces and related genera," Journal of General Microbiology, vol. 129, no. 6, pp. 1743-1813, 1983.

[30] F. Moosdeen, J. D. Williams, and A. Secker, "Standardization of inoculum size for disc susceptibility testing: a preliminary report of a spectrophotometric method," Journal of Antimicrobial Chemotherapy, vol. 21, no. 4, pp. 439-443, 1988.

[31] R. Cruickshank, J. P. Duguid, B. P. Marimon, and R. N. A. Swain, Medical Microbiology, Churchill Livingstone, London, UK, 12th edition, 1975.

[32] J. L. Krstenansky and L. Cotterill, "Recent advances in microwave-assisted organic syntheses," Current Opinion in Drug Discovery \& Development, vol. 3, no. 4, pp. 454-461, 2000.

[33] R. N. M. MacSween and K. Whaley, Muir's Textbook of Pathology, Edward Arnold, London, UK, 13th edition, 1992. 

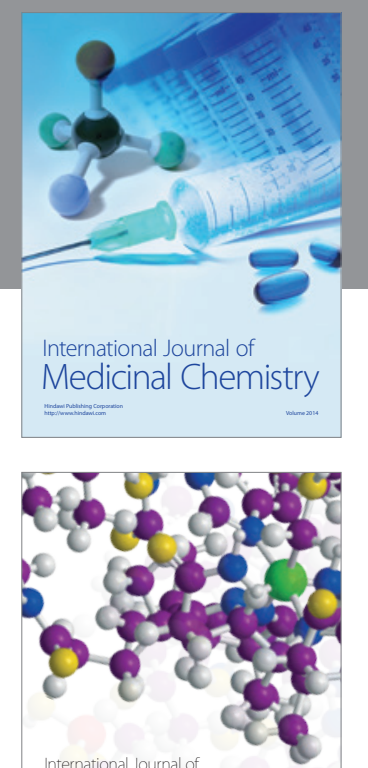

\section{Carbohydrate} Chemistry

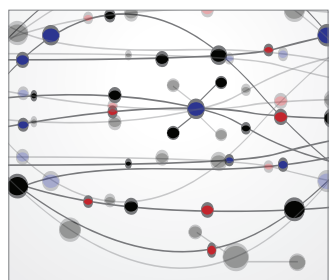

The Scientific World Journal
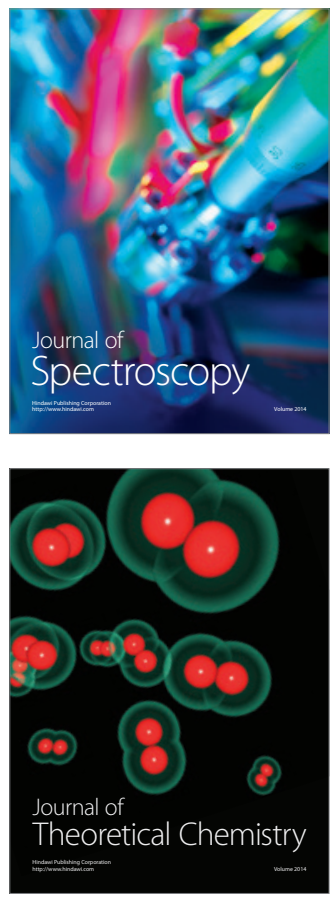
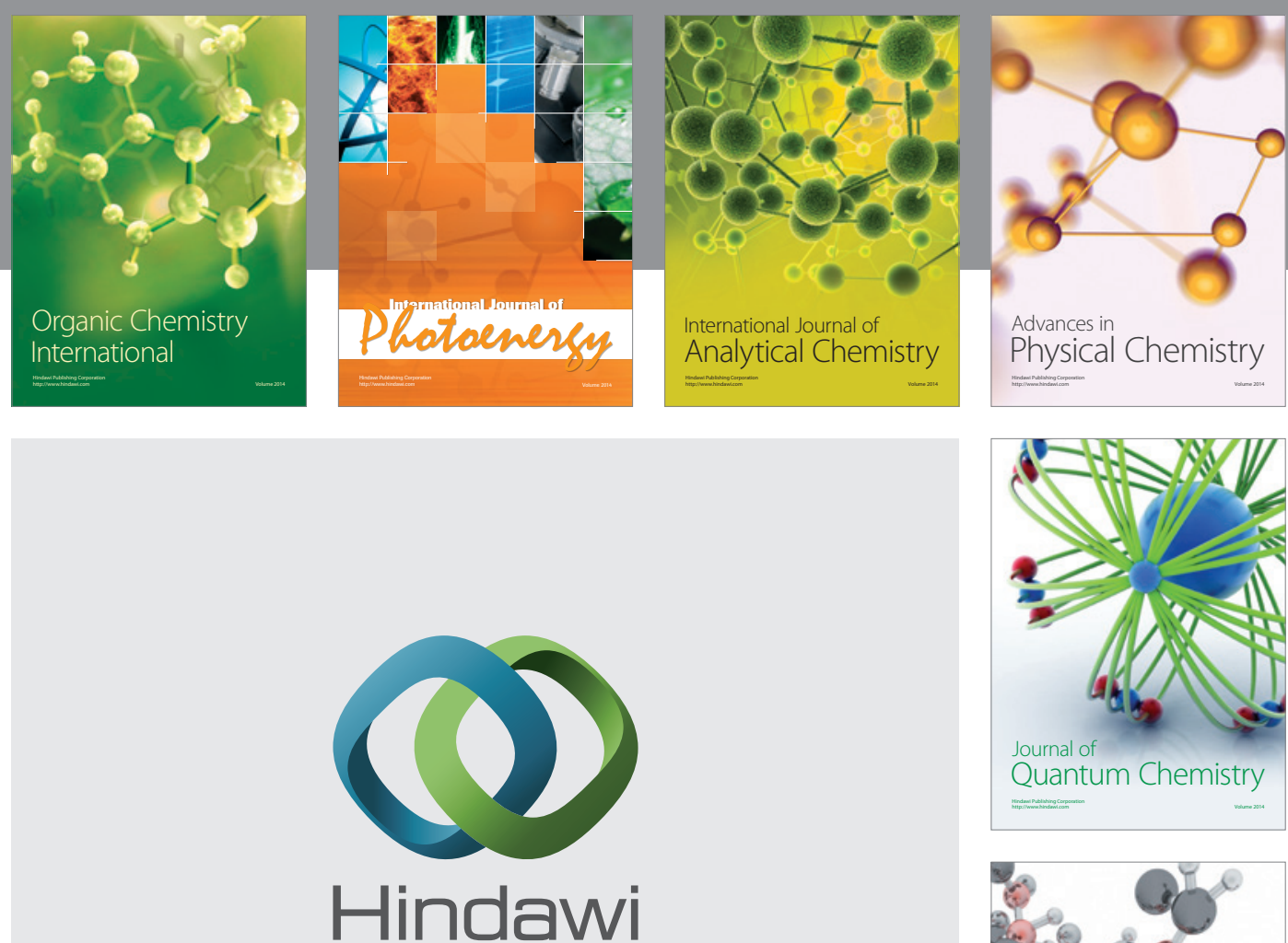

Submit your manuscripts at

http://www.hindawi.com

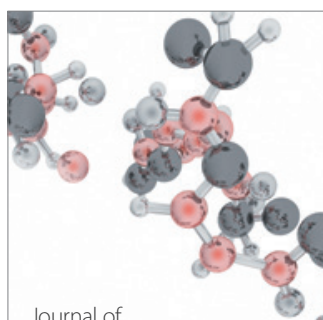

Analytical Methods

in Chemistry

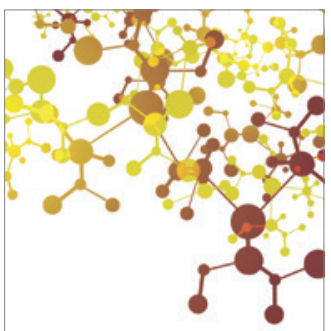

Journal of

Applied Chemistry

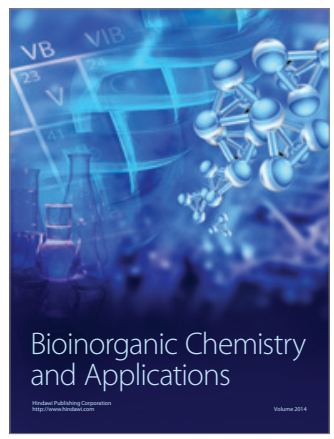

Inorganic Chemistry
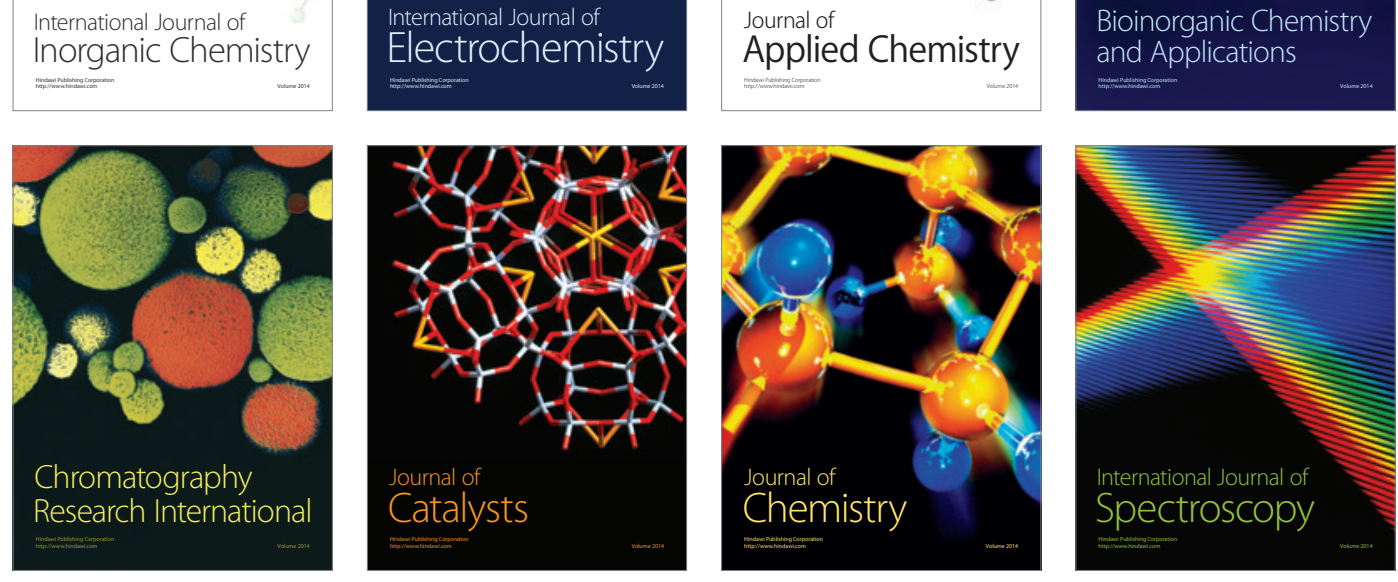